\title{
Analysis of Heart Rate Variability to Predict Patient Age in a Healthy Population
}

\author{
V. D. A. Corino ${ }^{1}$, M. Matteucci ${ }^{2}$, L. T. Mainardi ${ }^{1}$ \\ 'Department of Biomedical Engineering, Politecnico di Milano, Milan, Italy \\ ${ }^{2}$ Department Electronic and Information, Politecnico di Milano, Milan, Italy
}

\begin{abstract}
Summary
Objectives: To estimate age of healthy subjects by means of the heart rate variability (HRV) parameters thus assessing the potentiality of HRV indexes as a biomarker of age.

Methods: Long-term indexes of HRV in time domain, frequency domain and non-linear parameters were computed on 24-hour recordings in a dataset of 63 healthy subjects (age range $20-76$ years old). Then, as interbeat dynamics markedly change with age, showing a reduced HRV in older subjects, we tried to capture age-related influence on HRV by principal component analysis and to predict the subject age by means of a feedforward neural network.
\end{abstract}

Results: The network provides good prediction of patient age, even if a slight overestimation in the younger subjects and a slight underestimation in the older ones were observed. In addition, the important contribution of non-linear indexes to prediction is underlined. Conclusions: HRV as a predictor of age may lead to the definition of a new biomarker of aging.

\section{Keywords}

Age prediction, heart rate variability

Methods Inf Med 2007; 46: 191-195

\section{Introduction}

Biological markers of aging could be of great interest, as many old people develop conditions causing rapid or progressive disability before the end of their life and their biological age can be considered different from the chronological one. The ability to measure biological age may lead to a better prediction of the average rate of aging in the population and could be an important clinical tool in assessing both the risk for medical procedures and the likelihood of developing an early onset of age-related diseases.

It is a well-known fact that heart rate variability (HRV), explored by traditional methods in time and frequency domain and recently by methods based on chaos and non-linear theory [1-3], changes markedly from childhood to senescence [4]. HRV decreases with age, suggesting an age-dependent decline in autonomic nervous system activity in elderly subjects. This decline is evident in many time and frequency domain HRV parameters which are statistically and negatively correlated with age [4-6]. This trend has also been confirmed by non-linear methods.

Starting from the above considerations, some authors proposed models able to provide a simple description of the effect of senescence on HRV [7]. In this paper the possibility to capture the age-related influence on HRV is explored using principal component analysis (PCA) and neural networks. PCA is used to enhance single and independent contributions to the complex phenomena of HRV changes related to age, whereas the neural network is trained to predict patient age.

\section{Methods}

\subsection{HRV Features}

A set of 16 features were computed from the $\mathrm{RR}$ interval series. They include linear time and frequency domain parameters as well as non-linear metrics. They are briefly explained in the following.

\subsubsection{Time and Frequency Domain Methods}

Time domain and frequency domain indexes of HRV were computed on the entire 24-hour RR recordings [8]. Time domain parameters include the mean $(\mathrm{M})$ and the standard deviation (SD) of all normal RR intervals (NN intervals), the SD of the average NN (SDANN) calculated over 5-minute periods, and the mean of the 5-minute standard deviations of NN (SDNNi). In addition, the square root of the mean squared differences of successive RR intervals (rMSSD) and the percentage of interval differences of successive RR intervals greater than $50 \mathrm{~ms}$ (pNN50) were computed.

The power spectrum densities (PSD) were estimated on the entire 24-hour recordings. A smoothed PSD was obtained by averaging the rough PSD in 256 frequency bins. Logarithms of spectral power were evaluated in four pre-defined frequency bands [8], namely ultra-low frequency (ULF), very-low frequency (VLF), low frequency (LF) and high frequency (HF). In addition, the slope of the power spectrum ( $\beta$ slope) was calculated by a robust line fitting algorithm of $\log$ (power) and $\log$ (frequency) plot of the smoothed power spectrum over the frequency range of $10^{-4}$ to $10^{-2} \mathrm{~Hz}[9]$. 


\subsubsection{Poincaré Plot Indexes}

The Poincare plot is a scatterplot of the current RR interval plotted against the preceding RR interval. Points above the line of identity indicate RR intervals that are longer than the preceding RR interval, and vice versa. Accordingly, the dispersion of points perpendicular to the line of identity reflects the level of short-term variability (SD1) and it is quantified by the standard deviation of the distances of the points from the line of identity. The standard deviation of points along the line of identity reflects the standard deviation of the RR intervals, indicating the level of long-term variability (SD2).

\subsubsection{Approximate Entropy}

Approximate Entropy (ApEn), reflecting the likelihood that "similar" patterns of observations will not be followed by additional "similar" observations, is a measure quantifying the regularity of time series. A time series containing many repetitive patterns, i.e. a regular and predictable series, has a relatively small ApEn; a less predictable, i.e. more complex, process has a higher ApEn [2].

Given a sequence $S_{N}$, consisting of $N$ instantaneous heart rate measurements $(R R(1), R R(2), \ldots, R R(N))$ and a criterion of similarity $r$, an estimate of ApEn can be obtained by computing the following function:

$C_{i m}(r)=\frac{n_{i m}(r)}{N-m+1}$

The quantity $C_{i m}(r)$ is the fraction of patterns of length $m$ that resemble the pattern of the same length that begins at sample $i$, that is $p_{m}(i), n_{i m}(r)$ is the number of patterns that are similar to $p_{m}(i)$ [2]. We can calculate $C_{i m}(r)$ for each pattern $i$, and we define $C_{m}(r)$ as the mean of these $C_{i m}(r)$ values. The quantity $C_{m}(r)$ expresses the prevalence of repetitive patterns of length $m$ in $S_{N}$. Finally, we define the approximate entropy of $S_{N}$, for patterns of length $m$ and similarity criterion $r$, as

$\operatorname{ApEn}\left(S_{N}, m, r\right)=\ln \left[\frac{C_{m}(r)}{C_{m+1}(r)}\right]$ i.e., as the natural logarithm of the relative prevalence of repetitive patterns of length $m$ compared with those of length $m+1$. Standard [2] parameter values were chosen for the pattern length $(m=2)$ and similarity criterion $(r=0.2)$.

\subsubsection{Detrended Fluctuation Analysis}

The Detrended Fluctuation Analysis (DFA) quantifies fractal-like correlation properties of RR series [3]. Briefly, the interbeat interval time series of total length $N$ is first integrated and then divided into boxes of equal length $n$. In each box, a least-squared line is fit to the data and the integrated time series is detrended by subtracting this local trend $y_{n}(k)$. The root mean-square fluctuation of this integrated and detrended time series is calculated by

$$
F(n)=\sqrt{\frac{1}{N} \sum_{k=1}^{N}\left[y(k)-y_{n}(k)\right]^{2}}
$$

This computation is repeated over all time scales (box size) to relate $F(n)$ to the box size $n$. The slope of the line relating $\log (F(n))$ to $\log (n)$ is then computed in two scaling ranges: a short-range scaling exponent, $\alpha_{1}$, over periods of 4 to 16 beats, and a long-range exponent, $\alpha_{2}$, over periods of 16 to 256 beats [3].

\subsection{Principal Component Analysis}

The Principal Component Analysis (PCA) generates a new set of variables, the principal components $(\mathrm{PC})$, that result in a linear combination of the original ones [10]. All the $\mathrm{PC}$ are orthogonal to each other, therefore no redundant information is present. In this study, all 16 computed parameters are used in the PCA in order to understand the weight of each index.

\subsection{Feedforward Neural Network}

Artificial neural networks are generic nonlinear function approximators extensively used for pattern recognition, classification and non-linear regression $[11,12]$. A neural network is a collection of basic units, called neurons, computing a non-linear function of their input. Every input has an assigned weight that determines the impact this input has on the overall output of the node. In this paper, to improve generalization capability, we use the early stopping technique [13] consisting of using a validation set to stop the training algorithm before the network starts learning noise in the data as part of the model. The analysis is performed using a feedforward architecture and the Levenberg-Marquardt training algorithm [14, 15]. We use the early stopping technique to improve the generalization of the network; a validation set was obtained by hiding $25 \%$ of the data from the training set, the error on the validation is monitored during the training phase, and, as the error on the validation set begins to increase, the training is stopped. A two-layer network with three neurons in the first layer and one neuron in the second (output) layer is used; the transfer function is a hyperbolic tangent in the first layer and linear in the second one. In order to have more robust network results, we make the network run 100 times and then we take as a result the mean and the median of the 100 predicted ages for every subject.

\subsection{Experimental Dataset}

The measures of RR interval dynamics were computed from long-term recordings: 24-hour ECG recordings from 63 healthy subjects, 33 males and 30 females, of $55 \pm$ 15 years old (range 20-76) were analysed. Data come from the PhysioNet normal sinus rhythm RR interval database (47 recordings) and the PhysioNet normal sinus rhythm database (16 recordings) [16]. The RR interval time series were carefully reviewed to eliminate artefacts, premature, or missed beats. In particular the values during a gap resulting from artefacts, noises and exclusions of ectopic beats were assumed to be the linear interpolation of the preceding and the subsequent RR intervals. All subjects had at least 17 hours of ECG data, the average duration of the recordings was $22.5 \pm 1.5$ hours. The population was divided into two classes based on subjects' 
age: i) young, 20-50 years old and ii) elderly, $50-76$ years old.

\section{Results}

Relations among the first four components of PCA and the significantly correlated HRV indexes are reported in Table 1. The first principal component $(\mathrm{PCl})$ is mostly related to the traditional time and frequency domain parameters; in particular PCl appears highly correlated to SDNNi and spectral parameters, whereas the main contribu-
Table 1 The first four PCs and significantly correlated variables (in bold the most relevant)

\begin{tabular}{|l|c|c|c|c}
\hline & $\mathrm{PC1}$ & $\mathrm{PC2}$ & $\mathrm{PC3}$ & $\mathrm{PC4}$ \\
\hline $\log \mathrm{HF}$ & 0.31 & -0.17 & -0.10 & 0.15 \\
\hline $\log \mathrm{LF}$ & 0.29 & -0.11 & 0.31 & 0.19 \\
\hline $\log \mathrm{VLF}$ & 0.31 & 0.06 & 0.21 & -0.22 \\
\hline SDNNi & 0.34 & -0.01 & 0.15 & -0.07 \\
\hline SDANN & 0.17 & 0.47 & 0.02 & 0.21 \\
\hline ApEn & 0.19 & -0.41 & -0.09 & -0.12 \\
\hline$\alpha_{1}$ & -0.15 & 0.16 & 0.65 & -0.02 \\
\hline slope & -0.13 & 0.24 & -0.42 & 0.35 \\
\hline$\alpha_{2}$ & -0.09 & 0.28 & -0.33 & -0.55 \\
\hline $\mathrm{M}$ & 0.19 & 0.09 & 0.07 & -0.57 \\
\hline
\end{tabular}

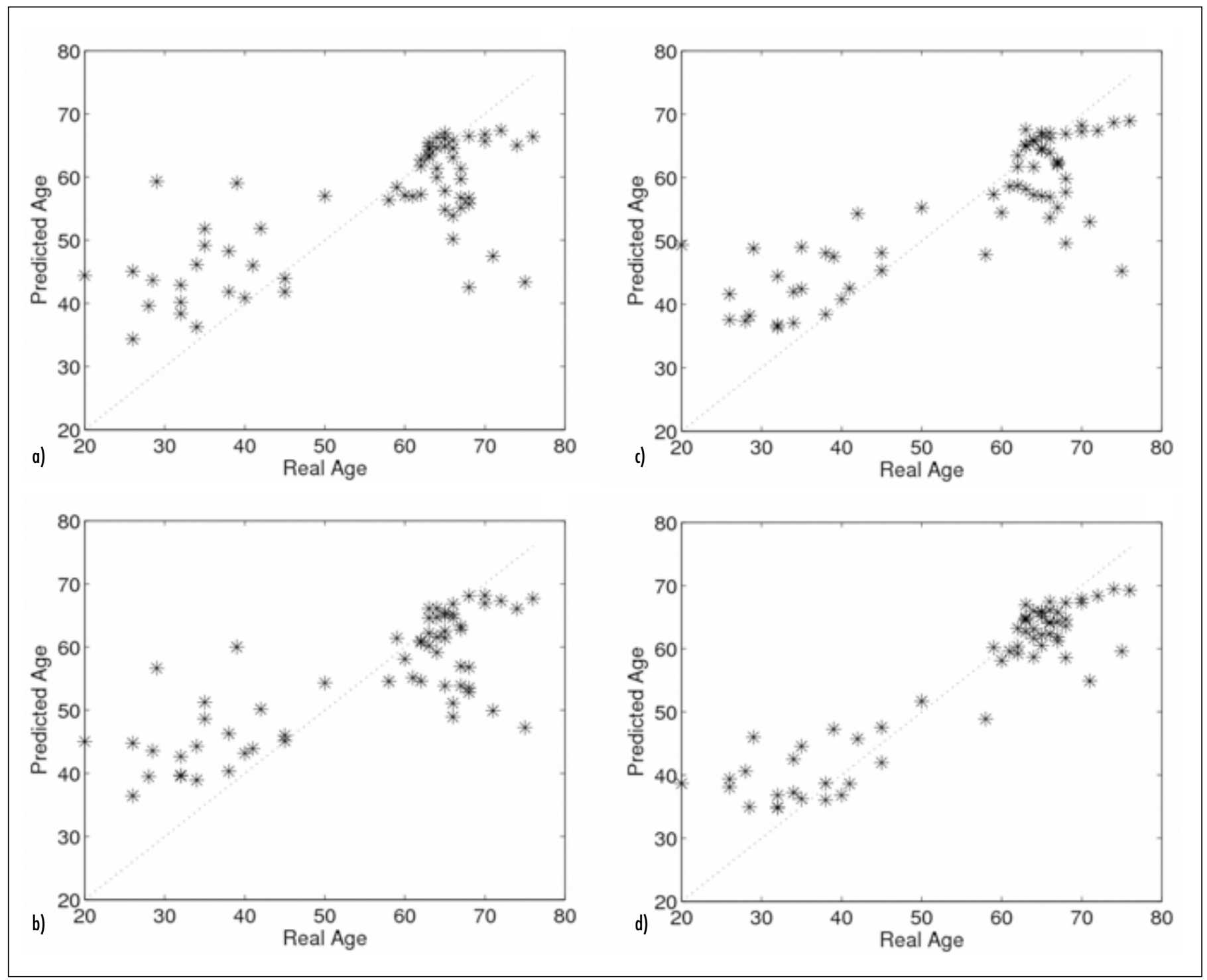

Fig. 1 Scatter plots of mean predicted age vs. real age, keeping a) $90 \%$, b) $95 \%$, c) $99 \%$, and d) $100 \%$ of variance. A clustering of the predicted age around the line of identity (dotted line) may be observed passing from a) to d). 
Table 2 Kept variance and respective number of necessary PC. Pearson Correlation Coefficient computed between real age and mean and median predicted age

\begin{tabular}{|l|l|l|l|}
\hline $\begin{array}{l}\text { Kept } \\
\text { variance }\end{array}$ & $\begin{array}{l}\text { Number of } \\
\text { components }\end{array}$ & $\begin{array}{l}\text { Pearson } \\
\text { correlation } \\
\text { (mean) }\end{array}$ & $\begin{array}{l}\text { Pearson } \\
\text { correlation } \\
\text { (median) }\end{array}$ \\
\hline $90 \%$ & 3 & 0.721 & 0.712 \\
\hline $95 \%$ & 4 & 0.771 & 0.761 \\
\hline $99 \%$ & 6 & 0.827 & 0.820 \\
\hline $100 \%$ & 16 & 0.934 & 0.945 \\
\hline
\end{tabular}

tions to the second component are provided by the ApEn, negatively correlated with PC2, and by SDANN. The most important roles in the PC3 are played by the slope of the power spectrum, negatively correlated, and by $\alpha_{1}$, the short-range scaling exponent of DFA, whereas the PC4 results highly negatively correlated with the RR intervals mean and with $\alpha_{2}$, the long-range scaling exponent of DFA. As every component represents a single and independent contribution to the investigated complex phenomena, the observed composition gives emphasis to the fact that the non-linear parameters provide important and complementary information, independent of the traditional indexes.

Figure 1 shows the scatter plots of real age vs. the mean predicted age, obtained using different number of PCA components (3, 4, 6, all components) corresponding to the $90-95-99-100 \%$ of kept variance. A progressive gathering around the identity line (dotted line) is clearly visible, increasing the number of components, demonstrating that the feedforward neural network provides a better estimate when the number of ana- lyzed features is increased. A slight overestimation in the younger subjects and a slight underestimation in the older ones can also be observed. Despite the reduction of the dispersion around the line of identity with the increase of kept variance, the tendency to overestimate age in the young and underestimate age in the elderly remains. In agreement the Pearson correlation coefficient computed between the real age and the mean and median predicted one increases with the number of components (see Table 2). Using all components, the predicted age is highly correlated with the real one.

In Figure 2, the mean and the standard deviation of the difference between predicted and real age are represented for the young and elderly population. It may be noted that the age predicted in the young class is overestimated, whereas the one of the elderly class is underestimated, but also that this prediction error decreases obviously keeping a larger percentage of variance.

The same analysis was performed on males and females separately: the general trend is confirmed, even if these results should be confirmed with a more numerous subset of patients.

\section{Discussion and Conclusion}

Increasing age during adult life seems to be associated with a reduction both in overall HRV and in the complexity of interbeat dynamics. This loss of complexity may be due to both structural factors and functional changes. Both the traditional time and fre-

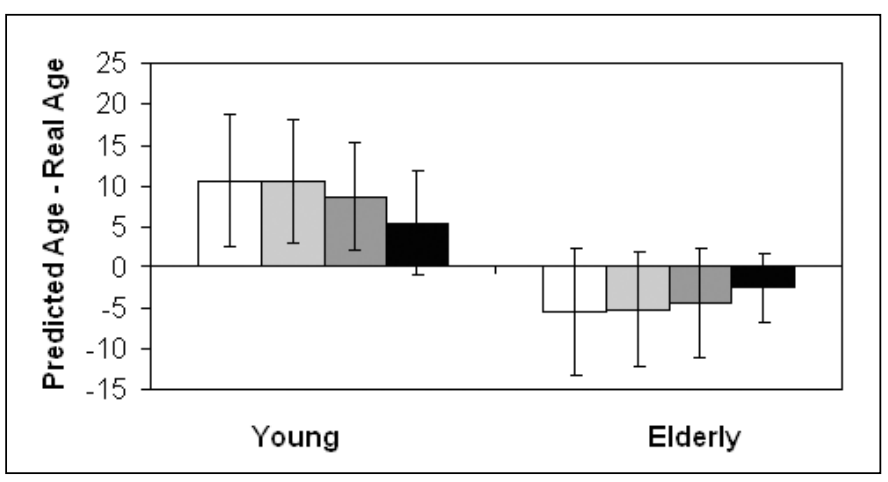

Fig. 2

Mean \pm SD of the difference between predicted and real age computed over the two groups of age (young and elderly), keeping 90-95-99 and $100 \%$ of variance quency domain and the recent non-linear analysis have shown that most HRV measurements were statistically and negatively correlated with age. In this paper, we were able to predict patient age in a healthy population, knowing HRV parameters: the correlation between mean predicted age and real age is higher than the results presented in literature [7]. This may be explained by the fact that non-linear parameters were included in the analysis and by the fact that that analysis was performed on the entire 24-hour recordings.

Assessing the performance of HRV as a predictor of age may lead to the definition of a new biomarker of aging and thus may be useful for screening purposes and for underlining cardiovascular differences between healthy young and adult subjects and healthy centenaries.

\section{References}

1. Bigger JT Jr, Steinman RC, Rolnitzky LM, Fleiss JL, Albrecht P, Cohen RJ. Power law behaviour of RR-interval variability in healthy middle-aged persons, patients with recent acute myocardial infarction, and patients with heart transplants. Circulation 1996; 93: 2142-2151.

2. Pincus SM. Approximate entropy as a measure of system complexity. Proc Natl Acad Sci USA 1991; 88: 2297-2301.

3. Peng CK, Havlin S, Stanley HE, Goldberger AL. Quantification of scaling exponents and crossover phenomena in nonstationary heartbeat time series. Chaos 1995; 5: 82-87.

4. Pikkujamsa SM, Makikallio TH, Sourander LB, Raiha IJ, Puukka P, Skytta J, Peng CK, Goldberger AL, Huikuri HV. Cardiac interbeat interval dynamics from childhood to senescence: comparison of conventional and new measures based on fractals and chaos theory. Circulation 1999; 100: 393-399.

5. Piccirillo G, Bucca C, Bauco C, Cinti AM, Michele D, Fimognari FL, Cacciafesta M, Marigliano V. Power spectral analysis of heart rate in subjects over a hundred years old. Int J Cardiol 1998; 63: 53-61.

6. Antelmi I, de Paula RS, Shinzato AR, Peres CA, Mansur AJ, Grupi CJ. Influence of age, gender, body mass index, and functional capacity on heart rate variability in a cohort of subjects without heart disease. Am J Cardiol 2004; 93: 381-385.

7. Colosimo A, Giuliani A, Mancini AM, Piccirillo G, Marigliano V. Estimating a cardiac age by means of heart rate variability. Am J Physiol 1997; 273: H1841-H1847.

8. Task Force of the European Society of Cardiology and the North American Society of Pacing and Electrophysiology. Heart rate variability: stan- 
dards of measurement, physiological interpretation, and clinical use. Circulation 1996; 93 : 1043-1065.

9. Sakata S, Hayano J, Mukai S, Okada A, Fucinami T. Aging and spectral characteristics of the nonharmonic component of 24 -h heart rate variability. Am J Physiol Regul Integr Comp Physiol 1999; 276: 1724-1731

10. Jolliffe IT. Principal component analysis (2nd edition). New York: Springer; 2002.

11. Bishop CM. Neural Networks for Pattern Recognition. Oxford University Press, Inc; 1995.

12. Haykin S. Neural Networks. A Comprehensive Foundation (2nd edition). New Jersey, USA: Prentice Hall; 1999.
13. Caruana R, Lawrence S, Giles CL. Overfitting in neural nets: backpropagation, conjugate gradient, and early stopping. In: Proceedings of Neural Information Processing Systems 2000. pp 402-408.

14. Marquardt D. An algorithm for least-squares estimation of nonlinear parameters. SIAM J Appl Math 1963; 11: 431-441.

15. Hagan MT, Menhaj M. Training feedforward networks with the Marquardt algorithm. IEEE Transactions on Neural Networks 1994; 5: 989-993.

16. The Normal Sinus Rhythm RR Interval Database and The MIT-BIH Normal Sinus Rhythm Database (http://www.physionet.org/physiobank/ database/nsr2db/).

\section{Correspondence to:}

Ing. Valentina DA Corino

Department of Biomedical Engineering

Politecnico di Milano

Via Golgi 39

20133 Milano

Italy

E-mail: valentina.corino@polimi.it 